\title{
Treatment-Emergent Adverse Event
}

National Cancer Institute

\section{Source}

National Cancer Institute. Treatment-Emergent Adverse Event. NCI Thesaurus. Code C142733.

A situation that emerges during treatment, having been absent pretreatment, or worsens relative to the pretreatment state. (ICH) 